In Situ

Revue des patrimoines
In Situ

Revue des patrimoines

$33 \mid 2017$

Patrimoine culturel immatériel et institutions patrimoniales

\title{
Midas au MEN
}

Midas at the MEN

\section{Olivier Schinz}

\section{(2) OpenEdition \\ Journals}

Édition électronique

URL : http://journals.openedition.org/insitu/15536

DOI : 10.4000/insitu. 15536

ISSN : 1630-7305

Éditeur

Ministère de la culture

Référence électronique

Olivier Schinz, "Midas au MEN », In Situ [En ligne], 33 | 2017, mis en ligne le 03 novembre 2017, consulté le 19 avril 2019. URL : http://journals.openedition.org/insitu/15536 ; DOI : 10.4000/ insitu. 15536

Ce document a été généré automatiquement le 19 avril 2019.

\section{(c) (i) () $\Theta$}

In Situ Revues des patrimoines est mis à disposition selon les termes de la licence Creative Commons Attribution - Pas d'Utilisation Commerciale - Pas de Modification 4.0 International. 


\title{
Midas au MEN
}

\author{
Midas at the MEN
}

\section{Olivier Schinz}

2 Ovide, dans le livre XI de ses Métamorphoses (85-193), narre l'histoire de Midas, roi des Phrygiens. Un jour, ceux-ci lui amènent le satyre Silène, ivre et perdu dans leurs contrées. En d'autres temps, Midas avait été initié par Silène aux orgies bachiques : heureux de le retrouver, il le raccompagne vers Bacchus qui lui propose alors de choisir sa récompense. «Fais que tout se change en or sous ma main! ». Ravi de ce nouveau pouvoir qui lui est immédiatement accordé, Midas ordonne de préparer un gigantesque buffet festif. Il se sert de vin : transformé en or ! Il saisit un beau morceau de viande grillée : transformée en or! Incapable d'ingérer quoi que ce soit, Midas regrette son erreur et demande pardon à Bacchus. Celui-ci accepte de revenir en arrière et enjoint Midas de se rincer dans le Pactole, rivière qui prend immédiatement une couleur dorée.

3 Fatigué des biens matériels de ce bas monde, Midas se réfugie dans les bois, auprès du roi Tmolus. La région abrite également Pan qui, un jour, a l'audace de se mesurer dans un tour de chant, accompagné de sa simple flûte de roseau, au brillant Apollon et à sa prestigieuse lyre incrustée de pierres précieuses et d'ivoire indien. Tmolus, choisi comme arbitre, désigne Apollon comme grand vainqueur. Midas ne l'entend pas de cette oreille et fait savoir qu'il prend le parti de Pan. C'est pour le punir de son audace qu'Apollon le châtie en sa partie coupable : ses oreilles, qu'il transforme en... oreilles d'âne.

Midas préserve sa réputation en cachant ses attributs métamorphosés et ridicules sous une tiare. Tout le monde ignore son châtiment. Tout le monde, sauf son coiffeur... Au bout d'un certain temps, celui-ci n'en peut plus d'être le seul dépositaire de ce secret important, dont il doit absolument se débarrasser en le partageant. Toutefois, désireux de rester fidèle à son maître, il creuse un trou dans le sable, crie de toutes ses forces son secret, puis rebouche le trou. L'année suivante, à l'endroit même où le trou a été creusé, poussent des roseaux. Agités par le vent, ceux-ci divulguent, au gré des courants, le secret si important : «Le roi Midas a des oreilles d'âne! Le roi Midas a des oreilles d'âne ! Le roi Midas a des oreilles d'âne !...». 
6 La Suisse a officiellement ratifié la convention de l'Unesco sur le PCI le 16 juillet 2008. Elle s'engage alors - comme le font tous les pays signataires - à réaliser un inventaire de son patrimoine culturel immatériel. Dès lors, deux mouvements conjoints vont aller de pair : d'une part, le processus de patrimonialisation lié à la réalisation de l'inventaire ; d'autre part, l'étude de ce processus.

7 Ce second mouvement est essentiellement le fait de chercheurs réunis sous une bannière unique : un projet de recherche lancé et dirigé par la professeure Ellen Hertz, de l'Institut d'ethnologie de l'université de Neuchâtel. Soutenu par un programme "Sinergia » du Fonds national suisse de la recherche scientifique, il se déroule en deux phases de trois ans chacune. La première, «Intangible Cultural Heritage : The Midas Touch? » de 2009 à 2012, la seconde "Intangible Cultural Heritage in Switzerland: Whispered Words » de 2012 à 2015․ Le Musée d'ethnographie de Neuchâtel est partie prenante de cette recherche, par l'intermédiaire de son directeur Marc-Olivier Gonseth, co-requérant. Son apport principal consiste en l'élaboration d'une trilogie d'expositions relatives à la question du PCI : Bruits (2010-2011), Hors-Champs (2012-2013) et Secrets (2015).

Nommé fin 2011 au MEN comme conservateur adjoint, j'ai participé à l'élaboration du troisième opus de cette trilogie. Mon point de vue est donc situé, plus encore que pour les deux autres, en son sein. J'utilise toutefois, dans ce texte, le «nous » également pour parler des deux premiers opus, lorsque je cherche à rendre le point de vue défendu par mon institution de rattachement.

93

Le PCI. Bruits. Hors-champs. Secrets. Le roi Midas. Comment tout cela se nourrit, se répond, tient ensemble ? À revenir a posteriori sur les trois expositions du MEN, j'ai parfois l'impression que les dieux se sont penchés pendant quelques instants sur la ville de Neuchâtel et sur son Musée d'ethnographie. Car, pour être très franc, ce que l'on peut raconter aujourd'hui de ces expositions n'était évidemment pas aussi clair en 2009, lorsque le premier projet de recherche s'esquisse. Chacun connaît l'excitation et l'incertitude de la période de montage de projet, toutes deux alors amplifiées par l'importance de la demande soumise au FNS par Ellen Hertz et les co-requérants. Les idées fusent, les contraintes rappellent à l'ordre, le temps manque. Dans cette effervescence surgit « The Midas Touch ». L'idée de tirer un parallèle entre Midas, son pouvoir magique et l'Unesco plaît, tant il est vrai que pour de nombreux acteurs, l'Unesco semble posséder cette capacité de transformer tout ce qu'il touche en or. Un label et la belle vie commence! Midas et son fameux coup de patte permettent, en une formule ramassée, évocatrice et efficace, de pointer un des enjeux essentiels que le projet de recherche se propose d'explorer.

11 Le MEN réfléchit parallèlement à un programme d'expositions qui pourrait accompagner ce projet. L'idée de ne pas suivre trop littéralement et directement le processus d'inventaire mais de proposer une forme de réflexion plus générale sur le patrimoine immatériel s'impose rapidement. C'est ici une histoire de tradition: si les liens entre recherche universitaire et exposition sont forts au MEN, ils ne sont jamais directs. Le Musée, pense-t-on, ne doit pas se contenter de présenter des recherches élaborées ailleurs mais doit être le lieu de production de nouvelles réflexions et de nouvelles connaissances. 
issions. Accords. Désaccords. Discussions. Finalement, trois titres sont choisis, comme un plan qui s'élabore sans que l'on sache encore très bien comment il sera mis en œuvre. Bruits. Hors-champs. Secrets.

\section{Alea jacta est!}

\section{4}

(fig. 1)

Figure 1

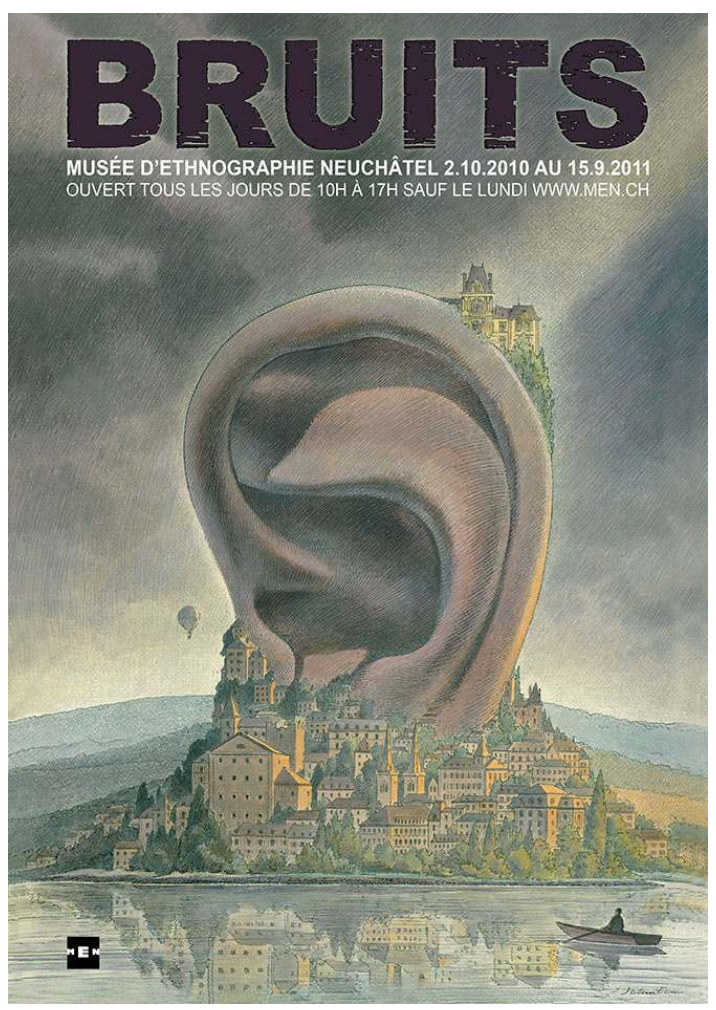

Bruits. François Schuiten.

(c) Musée d'ethnographie de Neuchâtel, Suisse.

Bruits : ce titre, au pluriel comme les deux autres de la trilogie du MEN, possède un rapport évident au patrimoine immatériel. Le bruit désigne péjorativement ce qui, au sein des productions sonores des sociétés humaines, n'est pas considéré comme suffisamment digne d'être patrimonialisé. Les enjeux sous-jacents à cette simple définition permettent d'entamer la réflexion du MEN sur le PCI, en s'inspirant de la malheureuse aventure du roi Midas : que se passe-t-il une fois que des productions sonores sont touchées par la grâce du patrimoine? Ou, pour prendre la question d'une manière un peu plus générale, quelles sont les carrières ${ }^{2}$ possibles des productions sonores ? Comment un son devient-il bruit ou patrimoine culturel immatériel ? Peut-il être l'un avant de devenir l'autre? Être l'un et l'autre à la fois ? Se modifie-t-il lorsqu'il est adoubé dans le monde du patrimoine ? Peut-il s'en échapper, ou y échapper?

Pour soulever ces questions, le visiteur est invité à suivre un parcours en cinq étapes, auxquelles viennent s'ajouter une introduction et une conclusion d'ordre poétique ${ }^{3}$. «Le souvenir de la houle », espace introductif, le fait passer sur une plage de coquillages qu'il ne peut qu'écraser, produisant ainsi une première performance «bruitiste » tout en 
étant plongé, immédiatement, dans l'ambiance scénographique générale. Car c'est un sous-marin géant qui sert d'espace d'exposition : un Nautilus échoué (ce que l'on découvre plus tard, à la fin de la visite), dans lequel se trouvent les différents espaces d'exposition, comme autant de salles du sous-marin.

Le premier espace permet de découvrir « Le bruit des autres » :

Le dispositif évoque un chargement de marchandises provenant de tous les coins de la planète, une partie des caisses portant des initiales, un titre, un lieu et une date. [...] Lorsque le visiteur marche sur une caisse, des sons étranges s'en échappent. Ils cessent dès qu'il retire son pied. Liés à l'ethnomusicologie, au folklore, aux avantgardes bruitistes et aux musiques populaires, les extraits disposés de manière chronologique ont tous, à un moment ou à un autre, été considérés comme des bruits et font tous en réalité aujourd'hui partie de l'histoire de la musique ${ }^{4}$.

Cette première immersion dans le monde des productions sonores trouve son pendant théorique dans la salle des machines qui suit, titrée « Le murmure de la théorie ». Celle-ci

met en scène une forêt de bouches d'aération d'où filtre un murmure continu. Lorsque le visiteur se rapproche d'une bouche, un personnage apparaît et un extrait sonore se déclenche, rapidement suivi d'une explication concernant le contexte de composition, de découverte ou de collecte de l'extrait concerné 5 .

Le visiteur est ainsi en possession des premiers éléments explicatifs et interprétatifs qui lui permettent de donner sens aux carrières réussies de sons initialement considérés comme indignes de faire partie de l'histoire officielle.

$21 \quad$ (fig. 2)

Figure 2

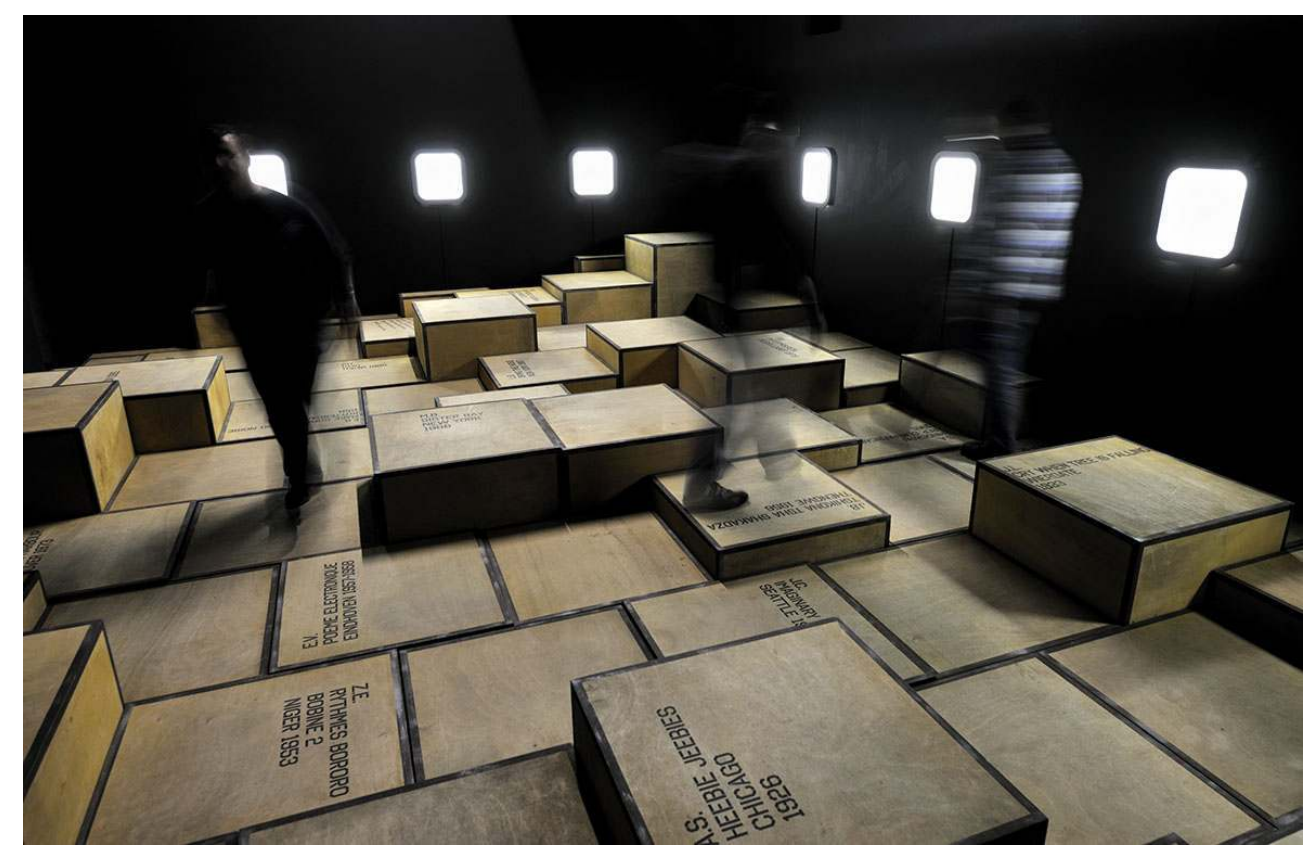

Bruits : le bruit des autres

Phot. Alain Germond. @ Musée d'ethnographie de Neuchâtel, Suisse.

Poursuivant sa quête personnelle, le visiteur pénètre, après qu'il a descendu quelques marches, dans une espèce de tour de contrôle et de surveillance intitulée « Les sirènes de l'urgence» : 
Dans la salle de contrôle, des écrans veillent sur un monde en danger, donnant l'alerte face à la disparition prochaine d'un chant, d'un rite, d'une langue, d'une pratique instrumentale, d'un répertoire de contes ou d'une technique artisanale. Et à travers le périscope, une vision d'Apocalypse semble leur donner raison ${ }^{6}$. largement, de l'inventorisation du patrimoine culturel immatériel (faire vite avant qu'il ne soit trop tard) et évoque, sur le mode de l'ironie, une posture fréquemment adoptée par les sciences humaines (celle de l'observateur inquiet). Quelques escaliers plus loin, le visiteur se retrouve au sein du plus grand espace de l'exposition, «L'écho des réserves ».

À la fois laboratoire de travail et salon, la médiathèque du sous-marin expose les collections audiovisuelles du MEN et permet au visiteur d'éprouver le poids de l'immatérialité ${ }^{7}$.

Et quel poids! Vastes collections ethnomusicologiques, appareils d'enregistrement, écrans, carnets de notes..., l'arsenal complet du parfait petit ethnologue de musée ne disparaît pas, comme par miracle, le jour où l'on s'intéresse à l'immatérialité du patrimoine.

(fig. 3)

Figure 3

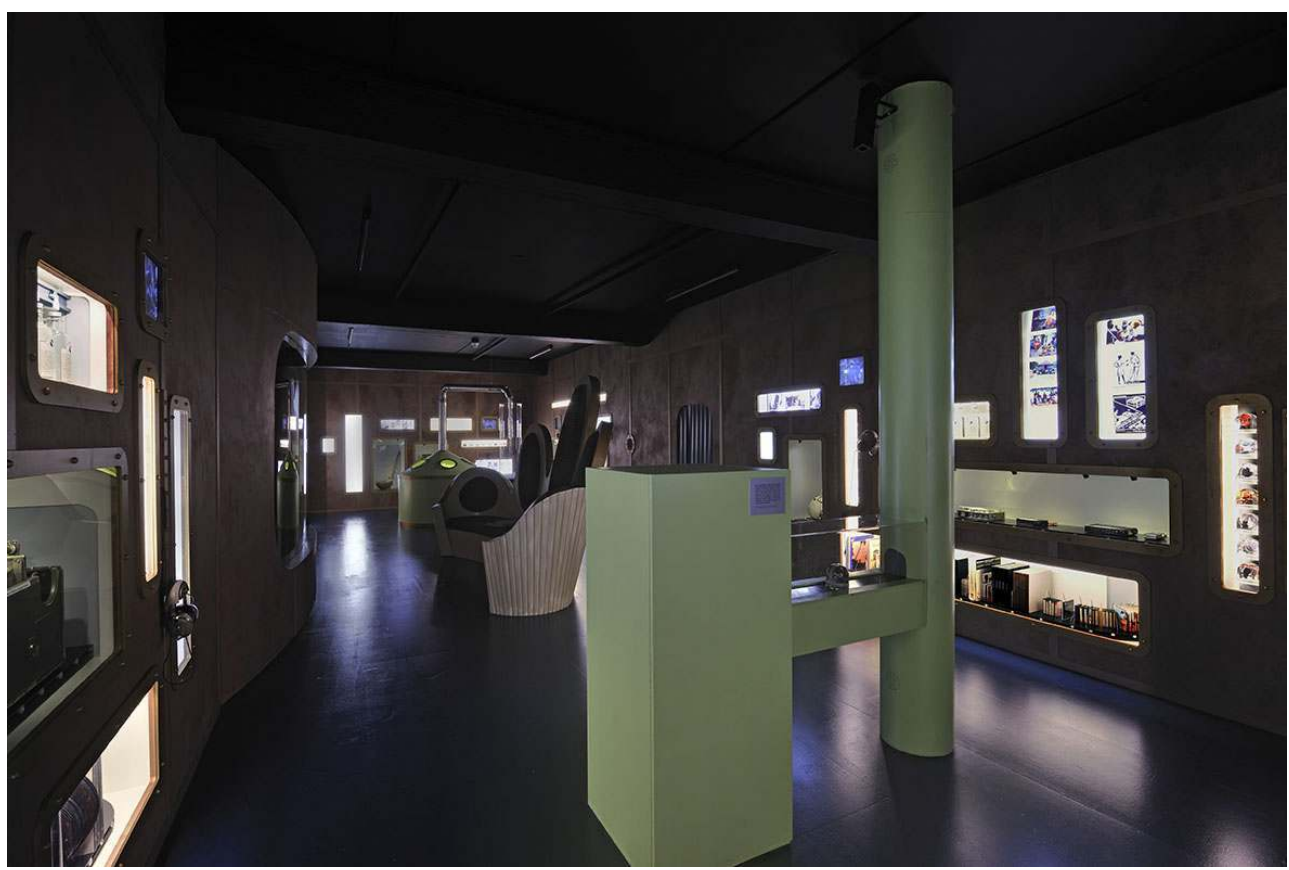

Bruits : l'écho des réserves. Au premier plan, le Trad'icer (de dos) et, au fond, le distillateur de collections.

Phot. Alain Germond. (c) Musée d'ethnographie de Neuchâtel, Suisse.

Que se passe-t-il lorsque des productions sonores sont rentrées dans les réserves des musées? Deux machines créées pour les besoins de l'exposition mettent en perspective des effets pervers possibles: le Trad'icer et le distillateur de collections. Le Trad'icer fabrique, à l'aide de données récoltées par des ethnologues sur le terrain, une série de boules à neige semblables à celles que l'on peut acheter dans les magasins de souvenirs du monde entier. La richesse vivante d'un patrimoine immatériel se réduit à l'image cliché, congelée, de la représentation pour touristes. Le distillateur de collections est, quant à lui, 
plus optimiste quant au devenir des biens rentrés dans les réserves de musées : il extrait la quintessence des productions sonores ou de leurs avatars matériels. Voici comment, dans un courriel, Marc-Olivier Gonseth me décrit son fonctionnement :

le dispositif active, grâce à un goutte-à-goutte qui semble provenir de la distillation d'une collection, différents programmes vidéo retraçant ici l'épopée d'un directeur de fanfare vaudois en Ethiopie. Dans un deuxième temps, ces extraits de distillation se transforment en thèses ou en hypothèses (selon le degré d'alcool de la cuvée, le Vaudois se retrouve à diriger la musique militaire de la Garde impériale d'Ethiopie, à la source du reggae en Jamaïque ou à l'origine du hip-hop à New York). Dans un troisième temps, on peut déduire que toutes les citations présentées sur les bouteilles sont issues du processus de distillation en question.

Il y a la cuvée " has been » de ceux qui ne jurent que par le passé, la cuvée «marchand de tapis " pour les amateurs de worldmusic divertissante ou les cuvées «technophile » et « technophobe » en fonction des accointances de chacun avec les supports permettant la reproduction de la musique. Au MEN, tous les goûts sont susceptibles d'être satisfaits.

Figure 4

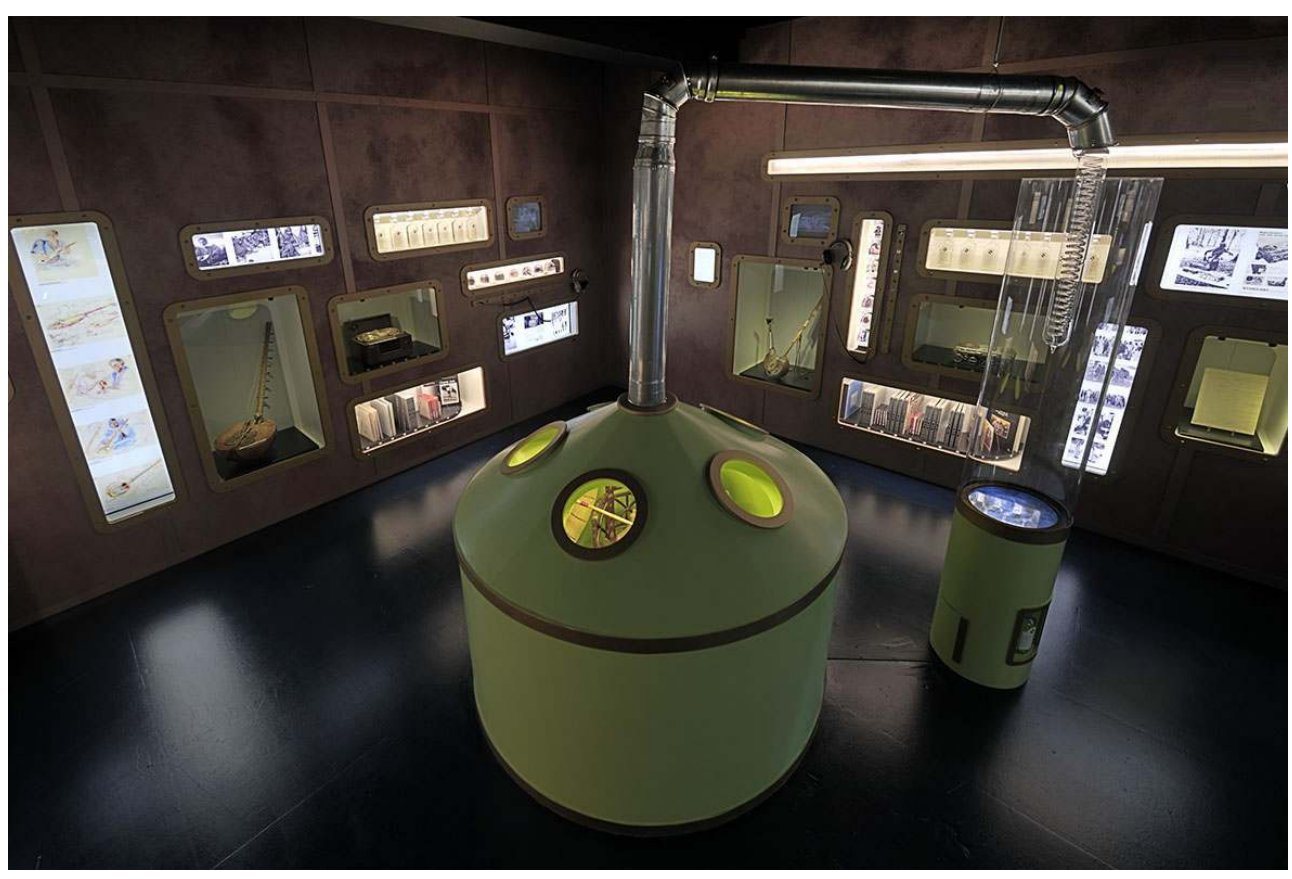

Bruits : l'écho des réserves. Le distillateur de collections.

Phot. Alain Germond. (c) Musée d'ethnographie de Neuchâtel, Suisse.

Puis il faut quitter les réserves, s'extraire du sous-marin. Le visiteur passe la porte de sortie et se retrouve sur une plage à moitié déserte. Il remarque que le sous-marin qu'il vient de quitter est en fait échoué sur cette plage. Mais dans "Le glouglou des pipelines ", l'espoir n'est pas mort.

Si la plupart des navires patrimoniaux s'échouent dans la nostalgie, le sentiment de perte et d'impuissance, d'autres acteurs envisagent avec un esprit plus créatif la reconfiguration du passé. Les données patiemment réunies par les spécialistes des arts et traditions populaires sont ainsi joyeusement piratées, détournées et intégrées à de nouveaux jeux culturels, économiques et sociaux ${ }^{8}$.

(fig. 5) 
Figure 5

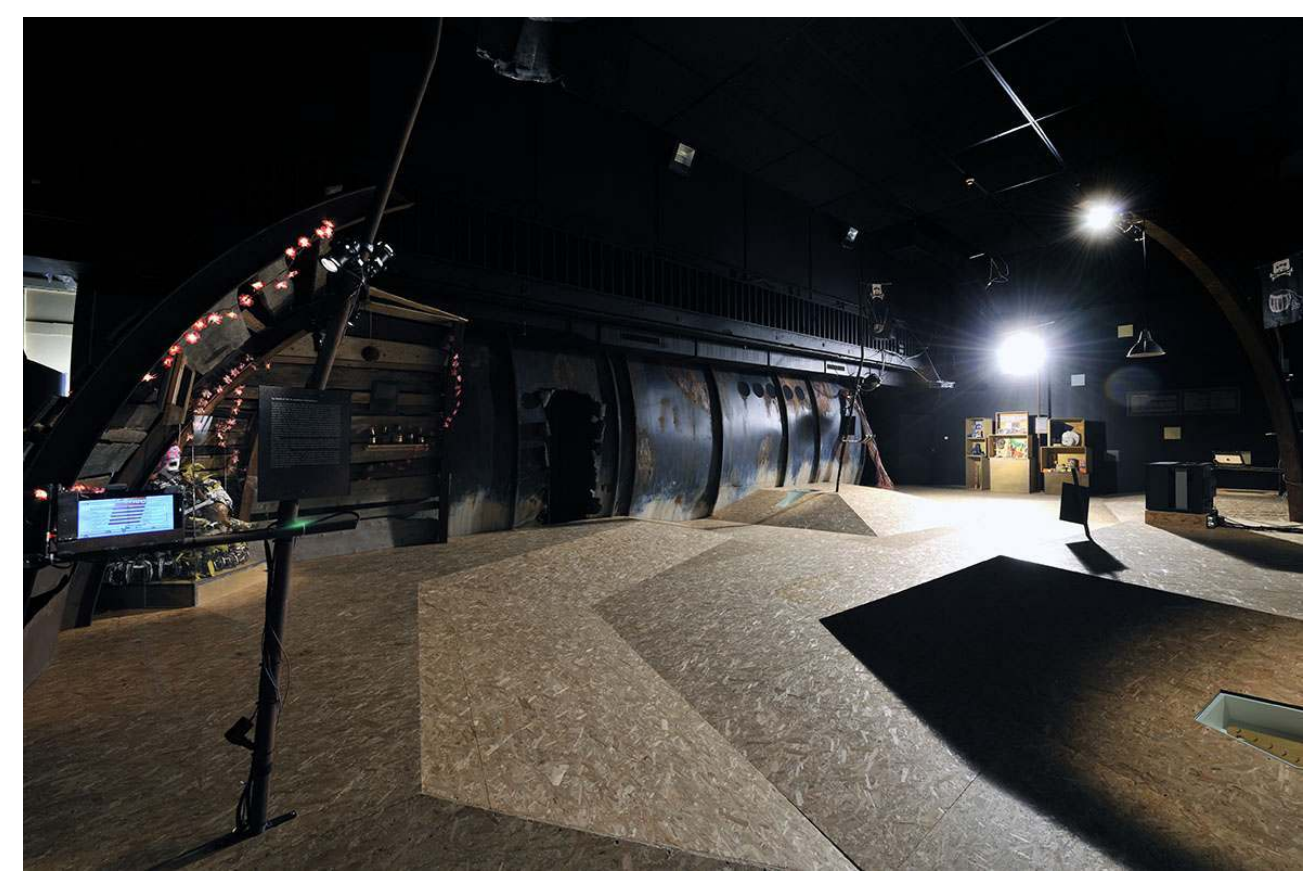

Bruits : le glouglou des pipelines.

Phot. Alain Germond. (c) Musée d'ethnographie de Neuchâtel, Suisse.

31 L'espace est un double hommage d'une part, à la vitalité des remix contemporains de sons patrimonialisés, et d'autre part, à Jean Gabus, conservateur du MEN de 1945 à 1978. C'est à lui que le MEN doit l'existence de la Black Box, le bâtiment dans lequel sont présentées les expositions temporaires qui fut inauguré en 1955. Le programme imaginé par Jean Gabus pour ce "Musée dynamique " était à l'époque extrêmement novateur: il osait lui adjoindre le terme de "Musée-spectacle». La salle nouvellement vernie possédait sa scène: ainsi, des danseurs brésiliens (1955), des marionnettistes balinais (1956) ou japonais (1969) ont proposé des spectacles au sein des expositions temporaires. Proposition avant-gardiste s'il en est, cette apparition de spectacles vivants dans l'espace consacré de l'exposition a, au fil des ans - et sans qu'aucune raison explicite connue ne soit avancée -, diminué en proportion jusqu'à s'éteindre complètement. Bruits a permis de ressusciter durant une année cette expérience grâce à une scène montée dans ce dernier espace, pas très loin de là où elle se trouvait à l'époque de Gabus.

C'est avec « Le fracas du ressac », proposition artistique projetée sur un rideau marquant la fin de l'exposition, que se termine le parcours des visiteurs. C'est surtout celui des productions sonores qui se termine au son de vagues dont on voit l'image projetée et que l'on traverse pour sortir de l'espace. L'extrait projeté est celui du Tempestaire du réalisateur Jean Epstein (1947).

On l'a vu, les productions sonores sont, comme tout objet ou toute chose de ce bas monde, susceptibles de poursuivre une carrière spécifique. Il y a des carrières réussies (un «bruit» qui devient une œuvre musicale reconnue), des carrières manquées, des carrières chaotiques ou des chemins tout tracés. De la création de la production jusqu'à son revival au sein de remix contemporains, tout un parcours semé d'embûches ou de 
passe-droits fait vivre et transforme une production initiale. La question de la patrimonialisation (ou de la labellisation) s'inscrit, de fait, dans cette problématique : elle influence directement la carrière des sons. Qu'il soit labellisé et sa carrière prend un nouvel essor - avec, bien évidemment, les risques de se fracasser sur certains des écueils évoqués dans l'exposition. Qu'il soit oublié par les instances officielles et ses chances de survivre s'amenuisent.

La mésaventure de Midas, incapable de manger ou de boire, puisque tout est transformé en or à son contact, nous met également sur une piste réflexive intéressante, parfois relayée par les acteurs du PCI : trop généreux, un label devient inefficace. À mesure que le nombre d'objets (matériels ou non) pris sous les ailes bienveillantes de l'Unesco - ou, à un niveau inférieur, dans les filets des inventaires nationaux - augmente, l'importance du label diminue. Trop de label tue le label. Lorsque tout est or, plus rien n'émerge d'un magma uniforme.

L'exposition Bruits pose ainsi des premiers jalons de réflexion autour du PCI. Elle énonce un parti pris essentiel du MEN pour l'ensemble de ses productions relatives à cet objet étrange : le musée ne doit pas être qu'un lieu de présentation d'objets largement traités et reconnus par les instances officielles de patrimonialisation, il peut également servir à questionner et à mettre en perspective le travail de ces instances. Certes, les musées peuvent tout à fait participer à la valorisation du PCI d'une région, d'un territoire spécifique, et élaborer des expositions mettant à disposition du public le résultat de travaux d'enquête ethnographiques. Mais il peut servir aussi à discuter de ce travail. À noter d'ailleurs que ces missions ne sont pas exclusives: Bruits ne propose pas qu'une critique des effets de la patrimonialisation des sons. C'est aussi l'occasion de présenter des sons, les approches théoriques qui permettent de leur donner du sens et le travail des différents experts à même de définir ces sons.

Bruits ferme ses portes le 15 septembre 2011. Le MEN propose alors un premier intermède à sa trilogie autour du PCI. "What are you doing after the Apocalypse? » s'installe dans les ruines scénographiques de Bruits. Ambiance fin du monde: le sous-marin vomit des flaques de pétrole, il se délite sous les assauts d'une Apocalypse annoncée.

(fig. 6) 


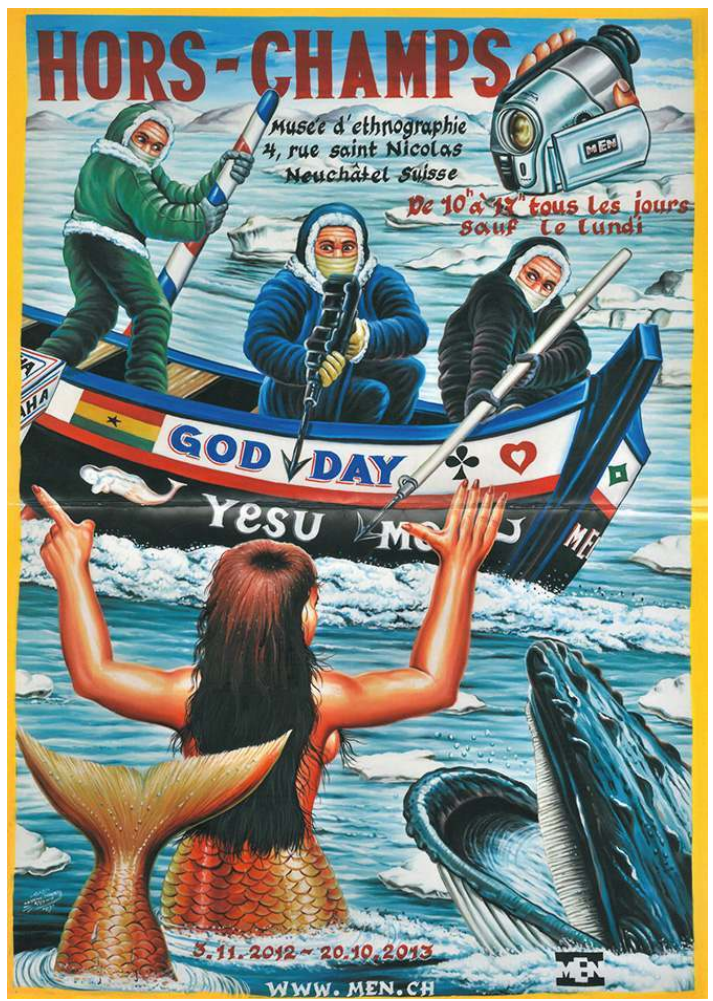

Hors-champs. «Heavy » Jeaurs et Regula Tschumi.

(c) Musée d'ethnographie de Neuchâtel.

C'est dans une atmosphère très différente que le visiteur est accueilli pour visiter Horschamps, à partir du 3 novembre 2012 : la salle d'exposition est - fait plutôt rare quoique loin d'être unique - entièrement dépourvue de cloisons et l'espace se laisse, a priori, entièrement embrasser du premier regard ${ }^{9}$. Le blanc domine. Parfois du bleu clair : une carte imaginaire du Grand Nord se déploie sous les pieds des visiteurs. Au milieu, des constructions de différents types. Certaines rappellent les habitats groënlandais, d'autres accueillent des modules d'expositions divers: vitrines, jeux, écrans multimédias, photographies, dioramas. Le curieux découvre des rideaux noirs, il les pousse, et il pénètre dans des petites pièces à l'atmosphère contrastée : tout y est sombre et noir. Il découvre tantôt un film d'Alain Cavalier, tantôt une série de photographies, tantôt un cercueil en forme de téléphone portable, tantôt un essai filmique. Personne n'est guidé au sein de cet espace, chacun y navigue librement.

Pour arriver là, le visiteur a dû traverser une pièce étrange : un magasin de réfrigérateurs qui, une fois ouverts, laissent découvrir des objets, des photos ou un jeu destiné à tous les visiteurs. C'est en ouvrant une dernière porte de chambre froide qu'il pénètre dans l'espace principal. Détail amusant: certains n'osaient, dans un respect muséal très incorporé, toucher les portes des réfrigérateurs, les ouvrir, et ne trouvaient ainsi pas l'entrée principale. De retour, après deux ou trois bonnes minutes de visite, pour demander à la réception s'il n'y avait pas quelque chose de plus intéressant à voir dans ce Musée...

(fig. 7) 


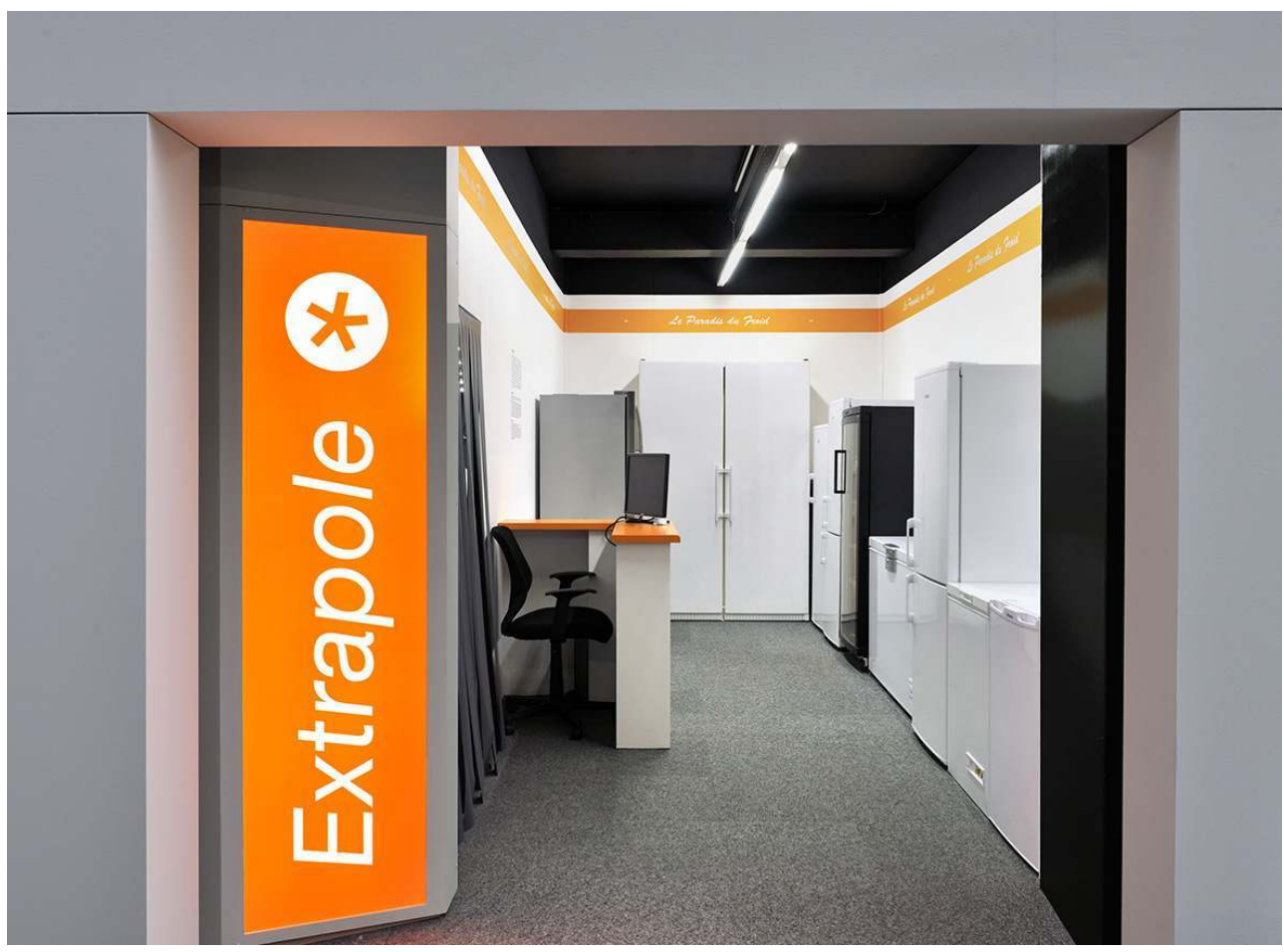

Hors-champs. Le magasin de réfrigérateurs. Une entrée difficile à trouver!

Phot. Alain Germond. (c) Musée d'ethnographie de Neuchâtel, Suisse.

La représentation est au cœur du questionnement de Hors-champs. Comment les musées d'ethnographie représentent-ils le monde qui les entoure ? Par des images, au sens large du terme: dessins, images analogiques, digitales, ou scénographies créées à partir d'expôts de tous types. Les scénographies sont considérées comme des images en trois dimensions au travers desquelles les visiteurs se promènent.

Parmi les objectifs poursuivis par l'utilisation des images dans les expositions ethnographiques, six sont mis en évidence. Les images permettent: (I) de classer (les objets et les personnes), (II) d'évoquer (le geste et le corps des absents), (III) d'esthétiser (les objets et les activités humaines), (IV) de représenter au plus près (une réalité fuyante), (V) de se remémorer (les acteurs et les événements) ou (VI) de provoquer (des chocs et des associations d'idées).

Finalement, car rien n'est jamais trop compliqué pour le MEN, ces objectifs peuvent être atteints par trois approches distinctes: (a) l'approche analogique, qui explore l'histoire des musées, (b) l'approche digitale, qui explore le développement des nouvelles technologies, et (c) l'approche poétique, qui explore l'association de l'art et de l'ethnographie.

47 Reprenons: le visiteur découvre des modules d'exposition en se baladant sur une carte imaginaire du Grand Nord. Dix-huit modules en tout, car chacun explore une des trois approches des six objectifs énoncés.

Six objectifs. Trois approches possibles pour chacun. Dix-huit modules.

(fig. 8) 


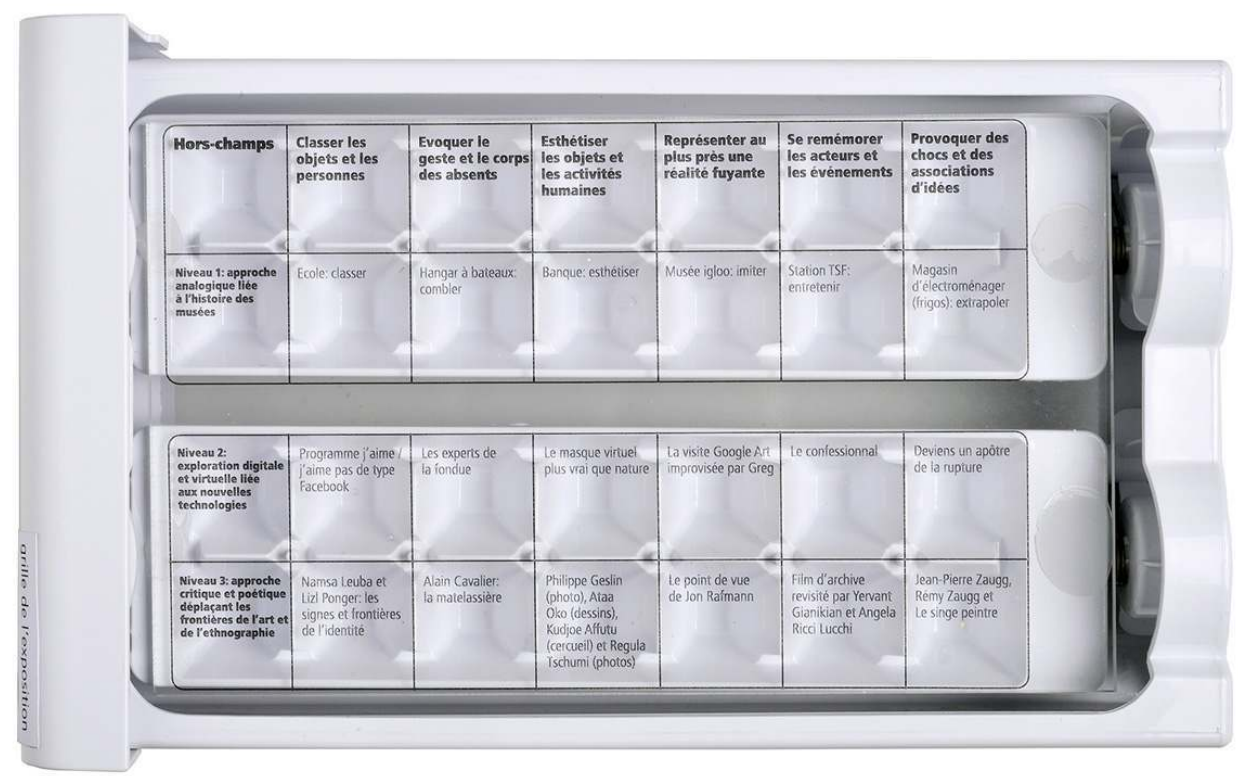

Hors-champs. Six objectifs, trois approches. L'exposition résumée dans un bac à glaçons...

Phot. Alain Germond. @ Musée d'ethnographie de Neuchâtel, Suisse.

Il y a là, par exemple, cet igloo inuit recréé au sein du Musée. Rien n'est inventé : il s'agit en fait de la recréation d'une recréation faite à partir d'une photographie. Jean Gabus, que le visiteur a déjà croisé dans Bruits, effectuait, en 1938, un terrain dans la baie d'Hudson. Un peu à la manière de M. Jourdain : jeune aventurier, journaliste, il part s'ouvrir au monde dans ces régions climatiques extrêmes et découvre l'intérêt de la découverte de l'altérité. Ce n'est qu'une fois rentré en Suisse qu'il décide de se former, à Fribourg, auprès du révérend père $S c h m i d t^{10}$ et qu'il obtient sa thèse de doctorat. Sur le terrain, il documente néanmoins, en faisant feu de tout bois, la vie quotidienne qu'il partage avec ses compagnons indigènes. Et deux ans avant de prendre une retraite bien méritée, il inaugure, en 1976 Les Esquimaux hier... aujourd'hui, exposition dans laquelle se trouve un igloo en carton-pâte (en fait, du polypropylène). Ainsi, la section « représenter » de Hors-champs imite cette imitation première. Par une mise en abîme, un objectif spécifique du Musée - la représentation de la réalité - est présenté ici par son approche analogique. 


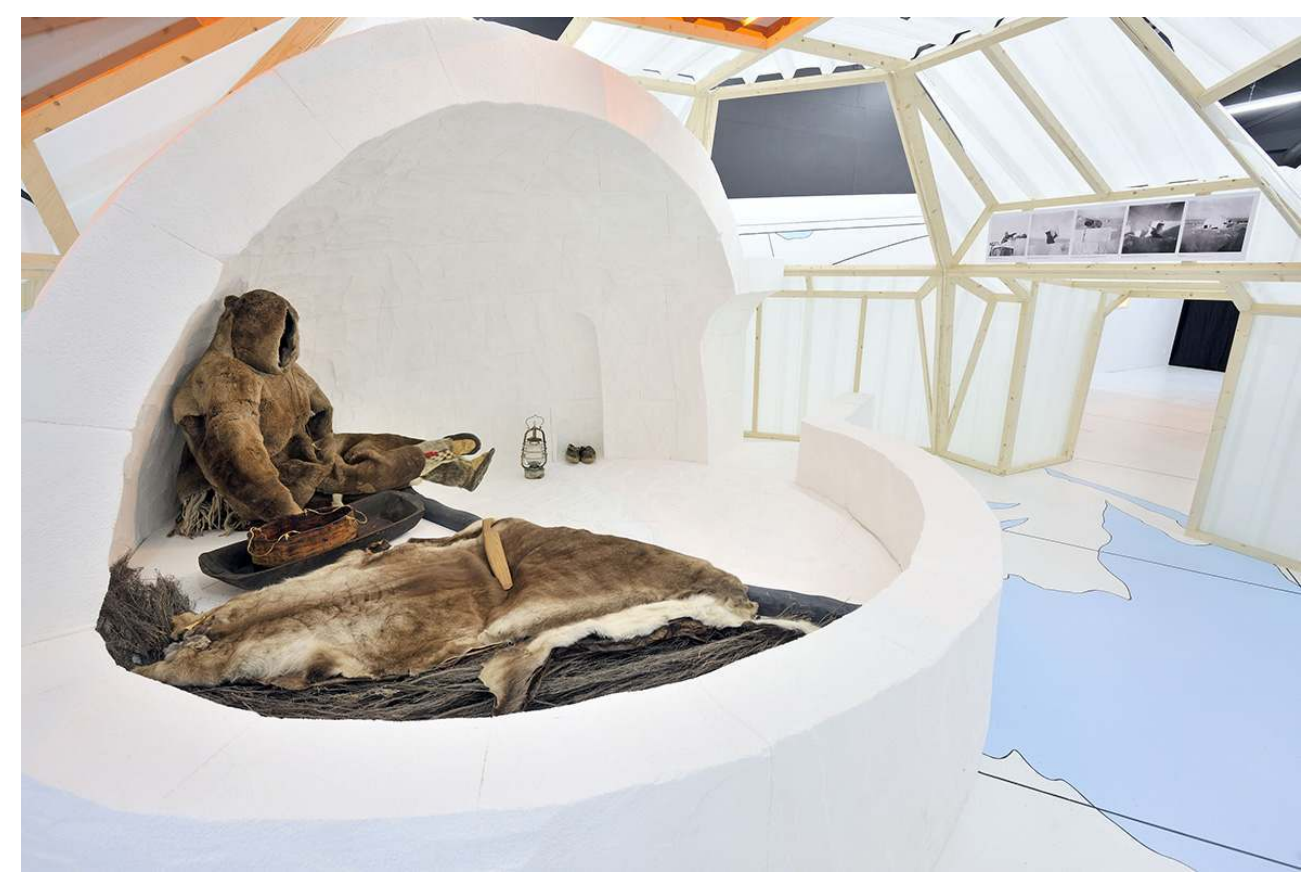

Hors-champs. Représenter. Approche analogique. Recréation d'un igloo inuit.

Phot. Alain Germond. @ Musée d'ethnographie de Neuchâtel, Suisse.

L'approche digitale - la deuxième approche - est évoquée par une visite «à la Google Street View » du MEN et de ses coulisses. Intitulée «Immersion », la section est réalisée par des moyens low-tech dans les murs du Musée. Le visiteur peut ainsi partir à la découverte des lieux qui lui sont habituellement inaccessibles - les réserves ou la cuisine notamment -, tout en choisissant son itinéraire favori. Gadget réifiant le fantasme d'un monde qui n'est plus cartographié ou représenté, mais entièrement dupliqué, et qui serait ainsi entièrement accessible depuis son écran, le module invite les visiteurs à mettre en question les attentes souvent exagérées exprimées autour des outils digitaux. La représentation, ici, se donne pleine, entière, complète, exhaustive. C'est l'abolition du hors-champ qui est (faussement) célébrée par ce type d'outils.

(fig. 10) 


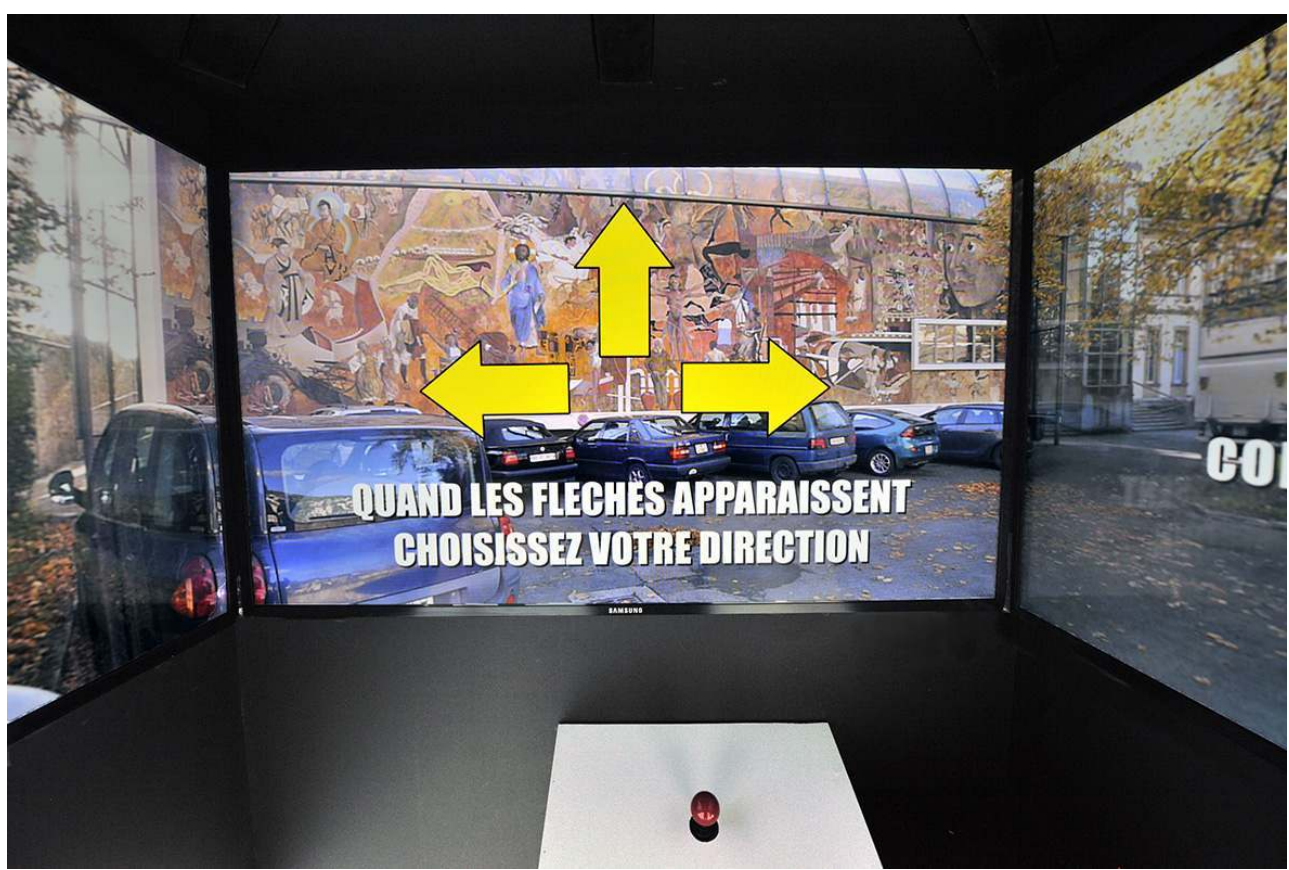

Hors-champs. Représenter. Approche digitale. Le MEN comme si vous y étiez.

Phot. Alain Germond. (c) Musée d'ethnographie de Neuchâtel, Suisse.

Le point de vue poétique ou artistique de cette section - la troisième approche - est illustré ici par les œuvres d'un artiste canadien, Jon Rafman ${ }^{11}$. Son long travail d'exploration des entrailles de Google Street View réintroduit du cadrage, du champ, dans l'immense base de données du géant américain. Jon Rafman se balade virtuellement aux quatre coins du globe et arrête son regard sur des scènes étranges, insolites, festives, intimes ou violentes captées par les yeux panoptiques de Google. Il en fait des captures d'écran qu'il reproduit ensuite, en grand format, dans des galeries d'art. Photographe sans appareil photo, cadreur d'une réalité préalablement saisie par les neuf yeux des caméras digitales de Google, Jon Rafman participe, à sa manière, à la réflexion élaborée sur la question de la représentation telle qu'elle est traitée dans les musées. 
Figure 11

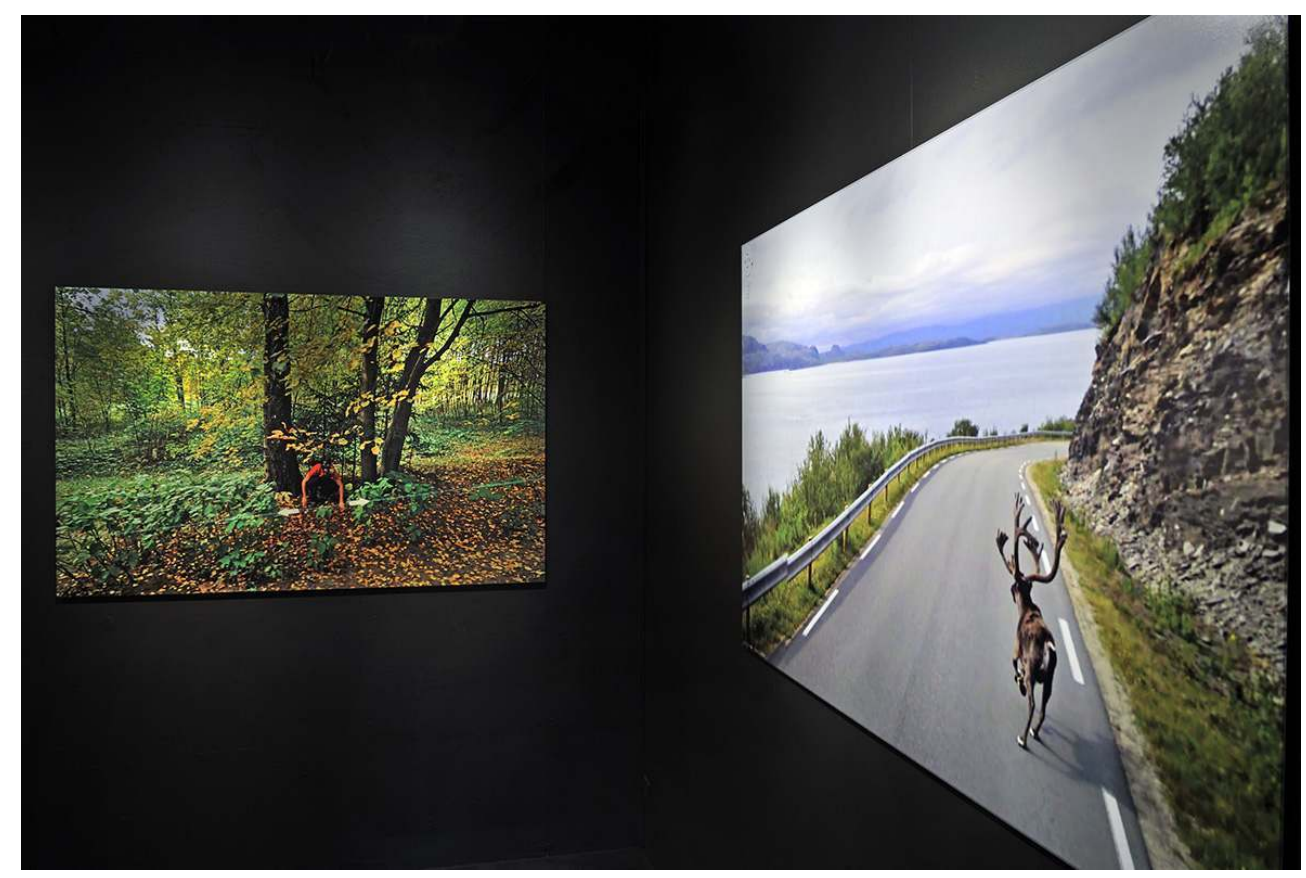

Hors-champs. Représenter. Approche poétique. Jon Rafman.

Phot. Alain Germond. (c) Musée d'ethnographie de Neuchâtel, Suisse.

Ces trois exemples d'approches distinctes d'un même objectif ("Représenter ») permettent de saisir la logique qui prévaut dans Hors-champs: une exploration extrêmement systématique de modes de représentations, une expédition au sein de laquelle le visiteur effectue son propre chemin et qui lui permet de voir, de saisir, d'expérimenter, d'analyser, de critiquer et de réfléchir à la question de l'angle, du point de vue, du regard imposé à toute personne qui pénètre dans un musée de société. Trois niveaux de réalités (trois approches) sont présentés car ils forment l'immense majorité si ce n'est la totalité - des approches possibles du monde par les musées de société. Tous les efforts et tous les talents du monde n'y pourront rien: chacune de ces approches exclut. Elle crée, à sa manière, du hors-champ : le regard cache, la vision voile.

Pan!

59 Aucun coup de feu mais, rappelez-vous, un concours. Les deux protagonistes sont, dans l'ordre, Apollon et sa prestigieuse lyre incrustée de pierres précieuses et d'ivoire indien, et Pan, accompagné quant à lui de sa simple flûte de roseau. Apollon doit gagner : il est l'élite, la musique savante, la réussite... Il faut être particulièrement téméraire, comme ce pauvre Pan, pour oser le défier à un concours de chant. Si l'affaire est dans le sac, pour ainsi dire, le concours permet de redonner une légitimité et une assise à Apollon. Le concours renforce les plus forts.

60 Et si les effets qui découlent de la patrimonialisation et des inventaires voulus par l'Unesco pour le PCI s'apparentaient à ce concours? Serait-ce exagéré de dire que les structures et processus mis en place pour désigner ceux qui méritent de faire partie d'une liste, ceux qui méritent une reconnaissance officielle ne sont pas extrêmement subversifs ? Très peu d'outsiders ont pris du galon à l'occasion des différentes opérations 
menées en Suisse - il en est sans doute de même ailleurs - et, à consulter les items présentés et retenus à l'Unesco, le doute n'a pas vraiment sa place : seul Apollon peut vaincre!

61 La mise en évidence (le regard, le point de vue) crée, je l'ai largement évoqué plus haut en présentant l'exposition, une forme de hors-champ. La mise en évidence, pour le dire autrement, abandonne. La sélection délaisse. L'inventaire rejette. 9

63 Fermée le 29 décembre 2013, Hors-champs laisse sa place à une seconde exposition intermédiaire. Imagine Japan ${ }^{12}$ présente le fonds Aimé Humbert : photographies, estampes, croquis, notes prises par le diplomate neuchâtelois (1819-1900) signataire du premier traité d'amitié et de commerce entre la Suisse et le Japon en 1864.

Pendant ce temps, dans les coulisses, se déroule une autre aventure, bien moins spectaculaire, mais tout aussi importante pour l'avenir du MEN. En mai 2012, la chambre législative de la Ville de Neuchâtel vote, à l'unanimité de ses membres, un premier budget de rénovation pour les bâtiments désuets du MEN. Pour des raisons diverses - raisons politiques et raisons de disponibilité budgétaire notamment -, ces fonds ne permettent que la rénovation d'une partie des bâtiments (la Villa, dans laquelle se trouvent les expositions permanentes), dans l'attente du vote d'un second crédit qui doit suivre. Les incertitudes politiques et opérationnelles nous interdisent, en 2013, de prévoir une exposition supplémentaire dans la Black box, sous peine de devoir soit raccourcir sa durée soit prolonger le délai des travaux. Il nous reste pourtant Secrets à réaliser, dernier opus du triptyque dévoué au PCI et à ses méandres. Que faire ? Sortir des murs, bien sûr... 10 


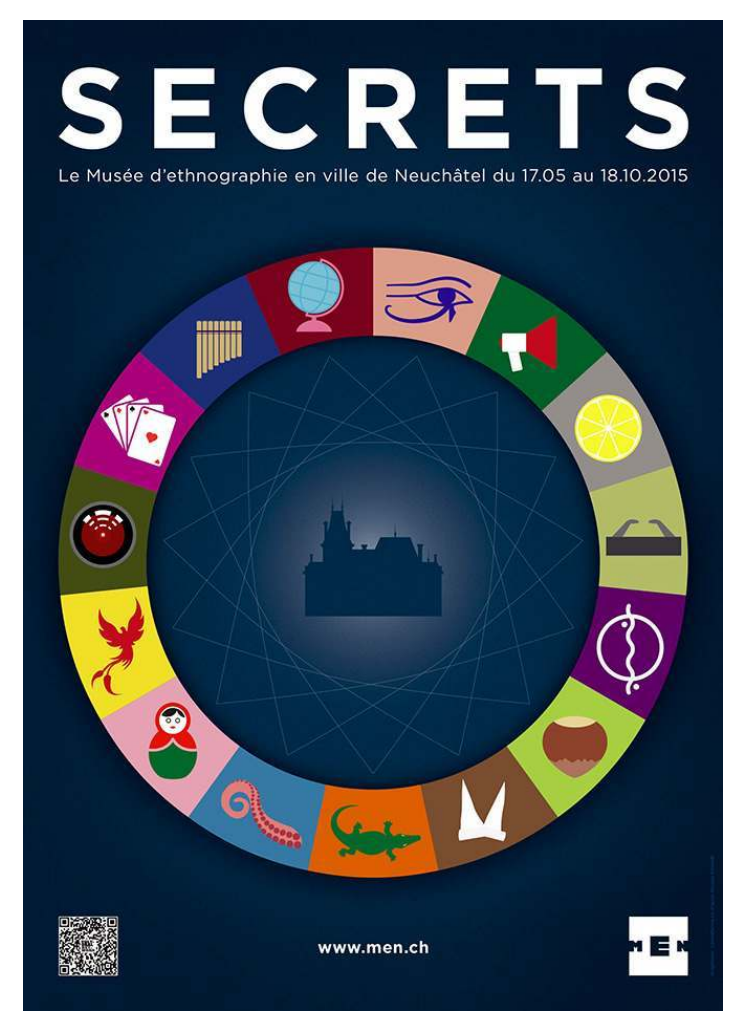

Secrets. Contreforme et Nicolas Sjöstedt.

(c) Musée d'ethnographie de Neuchâtel, Suisse. pictogrammes: une mappemonde, une tranche de citron, un tentacule de poulpe, une poupée russe, des cartes à jouer. Une flûte de Pan (tiens !), des oreilles d'âne (le lecteur de cet article peut imaginer quelque chose). Un porte-voix. Un phénix. Un crocodile. Et quelques autres encore, parfois difficiles à identifier de prime abord. Au centre, un bâtiment historique : la silhouette de la Villa, que beaucoup reconnaissent du premier regard. Des lignes relient le tout. L'adresse du site Internet du Musée invite à une première découverte car rien ne permet de savoir où commence «Secrets. Le Musée d'ethnographie en ville de Neuchâtel ». En naviguant sur les bonnes pages, le visiteur apprend que l'exposition débute à l'office du tourisme de la Ville, en plein centre, dans le même bâtiment que la poste principale.

70 Rendu sur place, un ticket d'entrée et un jeu de cartes lui sont délivrés. On lui demande de signer un engagement à respecter le secret de tout ce qu'il découvrira. De forme allongée ( $9 \mathrm{~cm}$ sur 23), les cartes ont un recto semblable - un jeu géométrique composé avec les mêmes pictogrammes que ceux de l'affiche - et un verso comportant des informations impossibles à comprendre: un extrait de plan de la ville, une phrase 
énigmatique (par exemple: «Avance le plus loin possible mais ne monte surtout pas les escaliers ») et, plus bas, un symbole. Toutes les cartes sont trouées, chacune à un endroit différent. Une carte (blanche) se distingue des autres. Elle donne les règles du jeu :

$\mathrm{Tu}$ es convié(e) à suivre un parcours composé de 15 lieux secrets en ville de Neuchâtel. Les cartes te permettent de t'orienter et te donnent des indices pour découvrir les différents sites. Sur chaque lieu, tu dois composter une carte pour te rendre au site suivant. La direction générale est donnée mais tu dois suivre les indices de la carte pour arriver à l'endroit précis. Attention: les symboles te permettent de composter la bonne carte au bon endroit. Si tu te trompes, tu ne pourras plus avancer. Tout au long de l'initiation, tu devras observer, lire, écouter et comprendre. Tu devras aussi faire preuve d'imagination, de créativité et de finesse d'esprit pour réussir à donner du sens aux 15 phrases et aux 15 objets/ pictogrammes auxquels tu seras confronté(e). À la fin du parcours, tu feras usage de ton expérience pour répondre aux questions posées. N’oublie pas que tu t'es engagé

(e) à ne divulguer à personne les lieux et les contenus découverts. Bonne chance!

71 À l'office du tourisme, une première scénographie présente, sous vitrine, la même rosace que celle de l'affiche. De vrais objets remplacent toutefois les pictogrammes. Le visiteur est prévenu : il doit « observer, lire, écouter et comprendre ", une invitation qui n'est pas vaine. Car dès à présent, son attention est requise. En comparant la rosace qu'il a devant les yeux avec celle de l'affiche, des esquisses de sens parviennent à son esprit. La relation entre les pictogrammes et les objets n'est pas toujours terme à terme : si le bonnet d'âne (en pictogramme) est bien un bonnet d'âne (en vrai), la tranche de citron se transforme en bouteille de jus de citron. Ce qu'il croyait être un crocodile se transforme en... pochette de DVD (Le Caïman, de Nanni Moretti). Premiers indices, premières pistes, premières interprétations. 


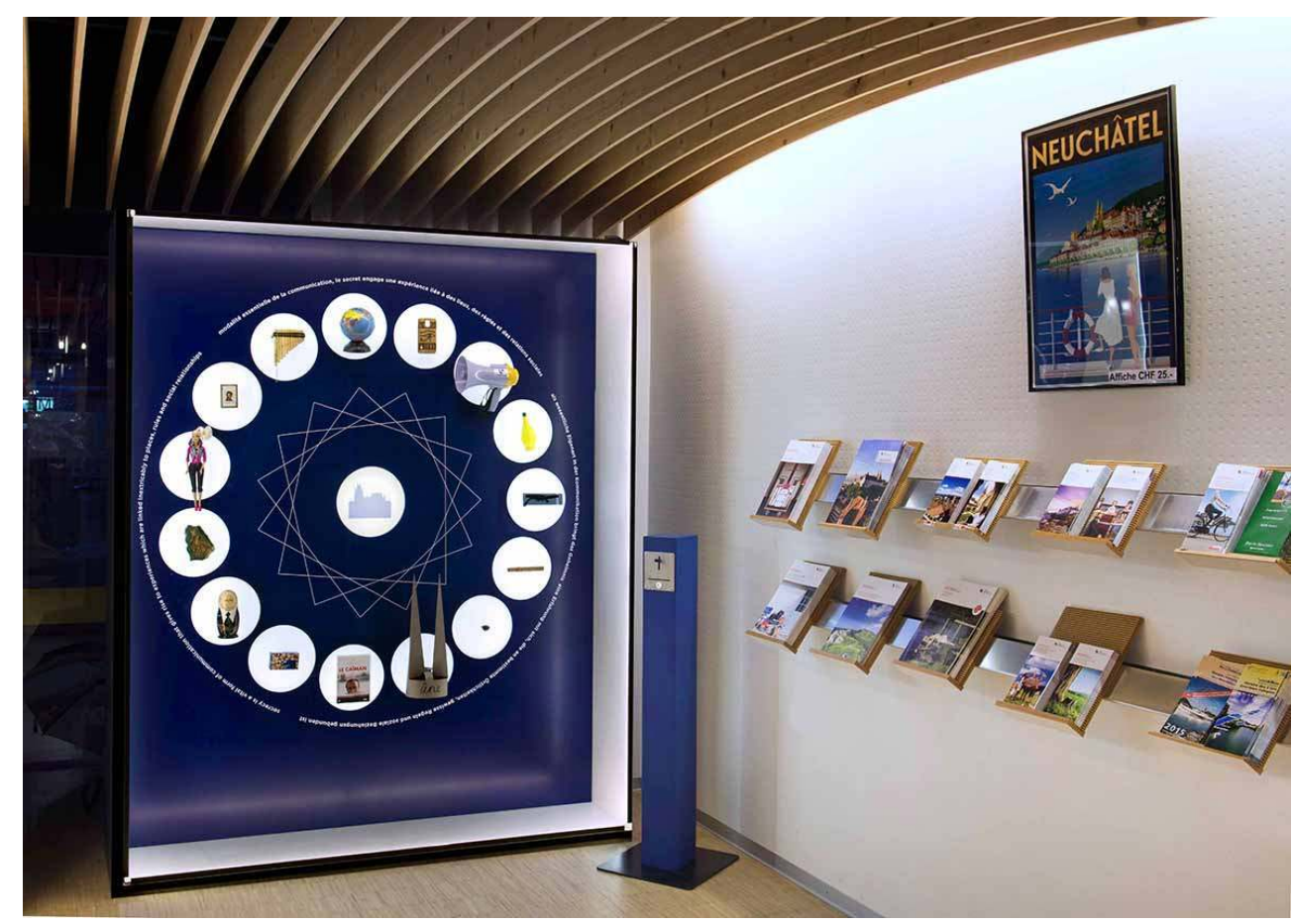

Secrets : l'office du tourisme. "Modalité essentielle de la communication, le secret engage une expérience liée à des lieux, des règles et des relations sociales ».

Phot. Alain Germond. (c) Musée d'ethnographie de Neuchâtel, Suisse.

Puis une phrase, imprimée autour de la rosace, s'ajoute à cette forêt de sens : «modalité essentielle de la communication, le secret engage une expérience liée à des lieux, des règles et des relations sociales ». Le jeu est lancé.

Lecteur : relis cette dernière section. Et imagine une manière de faire progresser ton interprétation. Pour t'aider, pense aux éléments que le visiteur avait à sa disposition : des cartes, des pictogrammes, des objets, une phrase... Nous t'offrons cela dans le désordre pour que tu puisses y mettre un peu d'ordre.

77 Profitons de cette pause pour une courte digression. Dans le processus habituel des relectures au travers desquelles est passé cet article, un relecteur (Christian Hottin) m'écrit ceci :

Ce qui est proposé dans l'article est bien une histoire, sous forme de récit : le fil est chronologique, les expositions intermédiaires sont intégrées au propos, la vie politique du canton apparaît en filigrane: le parti, original pour un article scientifique, pourrait être explicité en introduction.

La question soulevée par cette remarque me semble profonde, sans doute trop pour être pleinement discutée ici. J'hésite à suivre entièrement la proposition par laquelle elle se conclut. Voici plutôt une énigme supplémentaire : pourquoi insérer cette remarque ici dans le texte, et non en introduction, comme le suggère Christian Hottin? Quels parallèles à tirer entre une exposition qui se donne à découvrir sous la forme d'énigmes à résoudre, qui ne montre que des indices, et un article au " parti original »? 
Secrets. Une autre station, au hasard (ou presque). Le visiteur a quitté le centre-ville, il a déjà découvert une demi-douzaine de lieux, et les cartes l'invitent à monter sur les flancs du Jura, au jardin botanique. Une longue marche, sous un soleil qui fut souvent généreux en cet été 2015 . Il se retrouve dans un petit vallon, à l'abri des bruits de la cité, là où se nichent les espèces cultivées par les jardiniers de la ville. Un petit paradis.

Il découvre une haie de roseaux en plastique, sur lesquels est inscrit ce terrible secret, disséminé par le vent : "Le roi Midas a des oreilles d'âne ». L'indice marqué sur ses cartes l'invite à pénétrer dans un petit cabanon qui se trouve à côté. Il ouvre la porte et découvre cette sentence : "il se fraie irrésistiblement un chemin vers son destinataire ». Puis, au fond d'un puits, la reproduction d'un tableau aborigène connu, Water Dreaming at Kalipinypa, de Johnny Warangkula Tjupurrula. Le cartel raconte l'histoire de ce tableau, un tableau d'initié sur lequel des secrets sont, d'une certaine manière aussi, disséminés aux quatre vents.

(fig. 14)

Figure 14

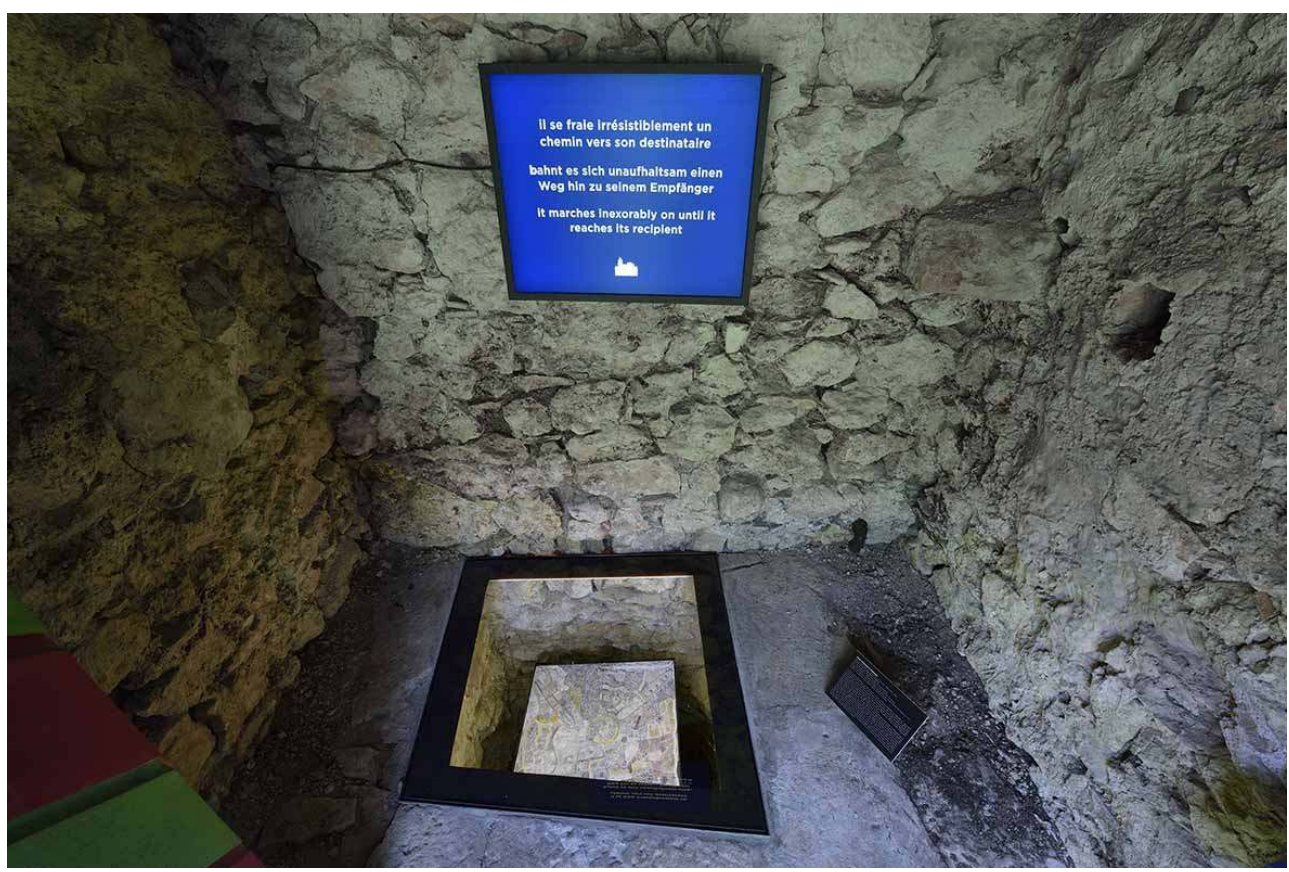

Secrets : le jardin botanique. «II se fraie irrésistiblement un chemin vers son destinataire ».

Phot. Alain Germond. @ Musée d'ethnographie de Neuchâtel, Suisse.

«Le secret est une modalité essentielle de la communication ». À associer au porte-voix, « il se fraie irrésistiblement un chemin vers son destinataire ». À associer aux oreilles d'âne, qui sont celles du roi Midas, dont le secret est inéluctablement arrivé vers ses nombreux destinataires, malgré les précautions prises par son coiffeur.

Ainsi se trame, lieu après lieu et par un jeu subtil d'associations, un texte dont le contenu final n'est autre qu'un condensé de théorie anthropologique sur le secret. Et puisque tu ne peux plus aller sur les lieux essayer de poursuivre ces jeux d'association, fidèle lecteur, 
voici le texte que les visiteurs ont eu la chance de reconstruire patiemment à mesure qu'ils progressaient dans leur initiation:

modalité essentielle de la communication, le secret engage une expérience liée à

des lieux, des règles et des relations sociales

présent dans toutes les sphères de la société

il structure aussi bien la vie publique que la vie privée

transmis par son détenteur et partagé par ses dépositaires

il se fraie irrésistiblement un chemin vers son destinataire

régulièrement décrié ou contesté (lorsqu'il s'agit du secret des autres), il est aussi

jalousement protégé et valorisé (lorsqu'il s'agit du secret des siens)

s'il contribue à brouiller les cartes et les rapports de force

il participe avant tout au maintien d'un ordre du monde

constamment mis en cause par les apôtres de la transparence

il réapparaît dès qu'une volonté politique ou une exigence sociale le menace

formidable ressort d'économies populaires, il suscite la curiosité, l'envie, le désir

il s'énonce dans des récits tentaculaires et polymorphes

qui tous produisent leurs héros et leurs figures de proue

à la fois proche et distinct du mystère

il dessine la carte d'un monde invisible qui se révèle à travers des indices, des symboles, des objets et des paroles.

L'écriture de l'exposition possède des points communs avec l'écriture d'un article scientifique. Il existe, assurément, autant de styles d'écriture dans l'un et l'autre domaine, comme il existe une doxa, des topoi, des ruptures aussi parfois. Depuis plusieurs décennies, nous avons choisi, au MEN, de faire des expositions comme on raconte une histoire : nous cherchons à entraîner le visiteur dans une balade active - tantôt très dirigée, tantôt très libre -, dans laquelle le visiteur doit s'ouvrir et fournir un effort d'interprétation, de décryptage, d'analyse sans lequel il passe à côté des questions que nous cherchons à soulever. Il peut exister une forme de naïveté, voire de vulgarité, dans l'idée que le musée est omniscient et que son problème principal est de réussir à vulgariser, à expliquer clairement, à médiatiser, à simplifier ou à expliquer son savoir au "petit peuple " (entendez, dans le langage courant, «faire des expositions tout public »). Nous préférons l'idée que le visiteur participe pleinement de l'analyse proposée par les stimulations que nous essayons de construire. Les récits que nous proposons sont situés : ils ont un auteur (une personnalité morale, bien souvent, le MEN), ils s'inscrivent dans un contexte particulier, ils ont une genèse, ils suivent des pistes, des routes, ils errent parfois, ils proposent des retours en arrière, des pauses, des culs-de-sac, ils sont dans la diachronie et dans la subjectivité. L'auteur de l'article dit «scientifique » peut, comme l'auteur d'exposition, choisir son camp : chercher à expliquer de manière didactique - et somme toute assez convenue - une réalité qu'il pense avoir pleinement saisie, ou chercher à entraîner le lecteur dans son histoire, dans sa genèse, le long des routes sinueuses empruntées.

La trilogie du MEN sur le PCI a sans doute quelque chose d'un ovni dans le paysage muséal traitant de la question du PCI. Si, à travers les trois thématiques choisies, il est difficile de nier que les questions du patrimoine et celle de l'immatérialité sont au cœur des problématiques soulevées, nous ne sommes pas non plus dans un traitement immédiat des items sélectionnés sur les inventaires régionaux ou nationaux. L'enjeu de ces trois expositions dépasse la présentation du PCI : il s'agit plutôt d'ouvrir la problématique, de proposer un traitement alternatif d'une question déjà largement étudiée de toutes parts. Le musée - une instance soumise à une évaluation, des contrôles et des routines 
différentes du milieu académique - peut jouer un rôle différent, plus ouvert aux jeux, à la critique, à la remise en question, aux intuitions et à une créativité libre.

Les musées d'ethnographie et autres musées de société possèdent une pratique plus que centenaire de la question du patrimoine et des enjeux afférents. Revisiter la question de la représentation, par exemple, peut se faire à l'aide d'une longue histoire, documentée, dont les musées sont les dépositaires. Représenter les Papous d'hier pose les mêmes questions que représenter les traditions gastronomiques françaises d'aujourd'hui. L'ensemble des problématiques traitées au sein des trois expositions du MEN présentées ci-dessus sont ainsi autant d'outils permettant la critique et l'analyse des processus mis en branle par l'Unesco depuis 2003.

\section{BIBLIOGRAPHIE}

GONSETH, Marc-Olivier et HERTZ, Ellen. « Quelques réflexions anthropologiques sur un territoire émergent ». Bulletin de l'académie des sciences humaines et sociales (Berne), 8/2, 2008, p. 38-41.

GONSETH, Marc-Olivier, KNODEL, Bernard, MAYOR, Grégoire et LAVILLE, Yann (dir.). Bruits. Échos du patrimoine culturel immatériel. Neuchâtel : Musée d'ethnographie, 2011.

GONSETH, Marc-Olivier, KNODEL, Bernard, MAYOR, Grégoire et LAVILLE, Yann (dir.). Horschamps. Éclats du patrimoine culturel immatériel. Neuchâtel : Musée d'ethnographie, 2013, 321p.

KOPYTOFF, Igor. «The cultural biography of things: commoditization as process ». The social life of things: Commodities in cultural perspective, 1986.

\section{NOTES}

1. - Ces deux projets, et surtout leurs résultats, sont présentés sur le site du FNS. L'ensemble de la riche bibliographie produite durant ces années de recherche y est disponible. Voir les sites : http ://p3.snf.ch/project-127570 pour la première partie du projet et http ://p3.snf.ch/project-141927 pour la seconde partie [consultés le 17/10/17].

2. - Au même sens que l'on parle de biographies et de carrières d'objets (voir KOPYTOFF, Igor. «The cultural biography of things: commoditization as process ». Dans APPADURAI, Arjun (dir.). The social life of things. Commodities in cultural perspective. Cambridge/New York: Cambridge University Press, 1986, p. 64-91, ici p. 66 sq.).

3. - Quelques photographies de l'exposition peuvent être trouvées sur le site Internet du MEN : http://www.men.ch/fr/galerie-de-photos/galerie-2010-2015/2010-2011-bruits/ [consulté le 17/10/17]. Le catalogue publié à l'occasion de l'exposition se compose de textes scientifiques sur la problématique et d'une riche présentation du parcours et des intentions des concepteurs (GONSETH, Marc-Olivier, KNODEL, Bernard, MAYOR, Grégoire et LAVILLE, Yann (dir.). Bruits. Échos du patrimoine culturel immatériel. Neuchâtel: Musée d'ethnographie, 2011). Finalement, un site Internet spécifique à l'exposition permet à tout un chacun de se replonger dans le parcours : http://www.expo-bruits.ch/ [consulté le 17/10/17]. 
4. - Les citations présentant les différents espaces sont issues du catalogue de l'exposition: GONSETH, Marc-Olivier, KNODEL, Bernard, MAYOR, Grégoire et LAVILLE, Yann (dir.). Op. cit. Ici, p. 16.

5. - Ibid.

6. - Ibid., p. 133.

7. - Ibid., p. 17.

8. - Ibid., p. 263.

9. - Le lecteur intéressé peut consulter le catalogue de l'exposition ou se reporter à son site Internet: http://hors-champs.men-expo.ch/. Des photographies d'exposition sont également disponibles sur le site : http ://www.men.ch/fr/galerie-de-photos/galerie-2010-2015/2012-2013hors-champs/ [consultés le 17/10/17].

10. - Wilhelm Schmidt (1868-1954), prêtre missionnaire, ethnologue et historien des religions, fondateur de la revue Anthropos.

11. - Dont on peut découvrir le travail en cours, exposé dans Hors-Champs, ici : http://9-eyes.com/ [consulté le 17/10/17].

12. - Voir le site: http://www.men.ch/fr/expositions/anciennes-expositions/black-boxdepuis-1981/imagine-japan/ [consulté le 17/10/17].

\section{RÉSUMÉS}

En nous contant l'histoire du roi Midas dans ses Métamorphoses, Ovide ne se doutait sans doute pas que, quelques siècles plus tard, sa narration allait servir le propos de trois expositions tenues entre 2010 et 2015 au Musée d'ethnographie de Neuchâtel (Suisse). C'est que les aventures à rebondissement de ce roi déchu et malheureux offrent une structure si riche qu'elles ont permis d'interroger, sous trois angles fort différents, certains impensés du patrimoine culturel immatériel (PCI). Bruits, Hors-champs et Secrets : trois explorations des échos, des éclats et des opacités du PCI. Trois exercices expographiques, trois expériences particulières qui décrivent, présentent, analysent, interrogent ou bousculent les processus de patrimonialisation mis en œuvre depuis la création de la convention sur le PCI par l'Unesco et sa ratification par la Confédération helvétique.

As Ovide recounted the story of King Midas in his Metamorphoses, he probably did not doubt that a few centuries later, his narration would serve the purpose of three exhibitions held between 2010 and 2015 at the Museum of Ethnography in Neuchâtel (Switzerland). The rebounding adventures of this fallen and unfortunate king offer such a rich structure that they made it possible to question, from three very different angles, some of the impensed of the intangible cultural heritage (ICH). Bruits, Hors-champs and Secrets: three explorations of echoes, shards and opacities of the PCI. Three expographic exercises and three specific experiments describing, presenting, analysing, questioning, or overturning the heritage processes implemented since the creation of the ICH Convention by Unesco and its ratification by the Swiss Confederation. 
INDEX

Keywords : Neuchâtel ethnography museum, exhibition, intangible cultural heritage, Métamorphoses

Mots-clés : Musée d'ethnographie de Neuchâtel, expositions, PCI, Bruits, Hors-champs, Secrets

\section{AUTEUR}

OLIVIER SCHINZ

Conservateur adjoint, MEN (Musée d'ethnographie de Neuchâtel, Suisse)

olivier.schinz@gmail.com 